\section{A gle $|\mathrm{d}| \mathrm{a}$}

\author{
In der Schweiz/En Suisse
}

\section{Woche/Semaine 20/2001}

16./17. Mai 2001

Frühjahrsversammlung der SGDV, Lausanne

Colloque de printemps SSDV, Lausanne

Thema: Pädiatrische Dermatologie

Sujet: La dermatologie pédiatrique

Prof. Daniel Hohl

Tél. 02131403 60, Fax 0213140382

E-Mail: Daniel.Hohl@chuv.hospvd.ch

\section{Woche/Semaine 29/2001}

18.-21. Juli 2001

8th World Congress on Cancers of the Skin, Zürich

PD Dr. R. Dummer + Dr. F.O. Nestle, USZ

Tel. +41 125525 07, Fax +4112554403

\section{Woche/Semaine 37/2001}

12.-14. September 2001

\section{Stratum corneum 3, Basel}

Kongress-Sekretariat: Annick Galmiche + Silvia Schweizer Pentapharm Ltd., Postfach, Engelgasse 109, CH-4002 Basel Tel. +41 61706 4848, Fax +41617064800

E-Mail: annick.galmiche@pentapharm.com www.stratumcorneum.ch

\section{Woche/Semaine 39/2001}

27-29 septembre 2001

\section{3e Réunion annuelle de la SSDV, Genève}

Président: Jean-Hilaire Saurat

Secrétaire scientifique: Liliane Didierjean

Clinique de Dermatologie, HUG,

$\mathrm{CH}-1211$ Genève 14

Tél. +41 2237294 41, Fax +41223729695

E-Mail: liliane.didierjean@hcuge.ch

\section{Woche/Semaine 47/2001}

23./24. November 2001

Dermato-allergologischer Kurs der SGDV, Bern

Prof. L.R. Braathen

Tel. +4131632 22 88, Fax +41313815815

\section{Im Ausland/A l'étranger}

\section{Week 25/2001}

June 19-24, 2001

2nd Joint International Psoriasis Symposium and European Congress on Psoriasis, San Francisco

Co-Directors:

Aldo F. Finzi, MD; Howard I. Maibach, MD;

Henry H. Roenigk, Jr., MD

Org.: Skin Disease Education Foundation, Chicago IL

Tel. 001312988 7700, Fax 0013129887759

E-Mail: sdef@sdefmail.com

June 22-24, 2001

SFRR 2001 - The Meeting of the Society for Free Radical Research Europe, Rome

Zeroseigongressi

Dr. Fabio Virgili

Via Benaco 15

I-00199 Roma

\section{Woche/Semaine 35/2001}

30. August-2. September 2001

Müller Cell Meeting 2001, Leipzig

Prof. Wiedemann

Universitätsklinikum Leipzig

Klinik und Poliklinik für Augenheilkunde

Liebigstrasse 10-14

D-04103 Leipzig

\section{Woche/Semaine 37/2001}

9.-14. September 2001

14th World Congress of the Union Internationale de Phlébologie, Rome

Dr. A. Mancini

G.C. Congressi

Via P. Borsieri 12

I-00195 Roma

\section{Week 26/2002}

June 27-29, 2002

10th International Conference on Behçet's Disease Berlin

Organisation: Prof. C.C. Zouboulis

Scientific Secretariat: Department of Dermatology

University Medical Center Benjamin Franklin

The Free University of Berlin, Fabeckstrasse 60-62

D-14195 Berlin (Germany)

Tel. +49 $3084456911 / 6910$, Fax +49 3084456908

E-Mail: zoubbereszedat.fu-berlin.de 